\title{
ROTATIONAL BEHAVIOR OF COMETARY-TYPE BODIES
}

\author{
ERIC: BOIS \\ Observatoire de la Côte d'Azur, Av. N. Copernic, F-06130 Grasse, France \\ and \\ PASCAL OBERTI and CLAUDE FROESCHLÉ \\ Observatoire de la Côte d'Azur, Le Mont Gros, B.P. 139, F-06003 Nice Cedex, France
}

\begin{abstract}
The present paper deals with a general dynamical qualitative study of the rotational motion for cometary-type bodies submitted to gravitational torques. Numerical experiments of the evolution of comet nucleus attitude have been then performed, including the Sun and Jupiter's disturbing torques in the model. Results show small effects of the solar gravitational perturbation for Halley-type orbits. Only a very close-approach with Jupiter induces notable effects. The latter configuration presents some interesting sensitivity to initial conditions.
\end{abstract}

Key words: comet nuclei, rotation, Eulerian oscillations, physical librations, close-approach

\section{Introduction}

Nucleus rotation is assumed to greatly influence solar exposure and thus gas ejection, or more generally, non-gravitational forces. Only gravitational disturbing effects are concerned here and have been investigated by numerical simulations. These preliminary works on dynamical features of the rotational motion for cometary-type bodies are required to understand the basic mechanisms. They are developed in [1]. First, some results on the "free" rotation in space are exposed, then the effects of solar perturbations are studied, and finally some close-approach configurations with Jupiter are investigated.

Two different angular sequences are used to locate a body-fixed rotating frame $(O, x, y, z)$ relative to a fixed reference frame $(O, X, Y, Z)$, both with the origin at the center of mass: 3-1-3, and 1-2-3. Numbers 1,2 , and 3 refer respectively to the axes $x$ or $X, y$ or $Y, z$ or $Z$. Shifting rules can be found in [2]. The two sequences are of different types, and the singularities occur in different configurations. However, after the integration has been carried out, the motion is only described in the classical 3-1-3 sequence (precession $\psi$, nutation $\theta$, proper rotation $\varphi$ ), making it easier to interpret the results. Throughout the paper, the comet nucleus is modeled by an ellipsoid, its three axes of inertia being chosen with the following order: $a>b>c$. The rotation of greatest energy is applied around $c$. For every figure, the $\varphi$-angle is cleared out of its mean rotation.

\section{Free rotation in space}

The following curves represent the rotational motions of cometary-type bodies (shape, periods and orientation) without the action of disturbing torques. In this case, the three angular momentum components remain constant in the fixed frame. The non-perturbed rotation of a body with three unequal axes of inertia is expressed using Jacobi elliptic functions. With only one non-zero initial angular velocity, the solution is simply reduced to a linear function without any libration. Figures 1 and 2 show some examples of Eulerian oscillations obtained for two sets of initial 

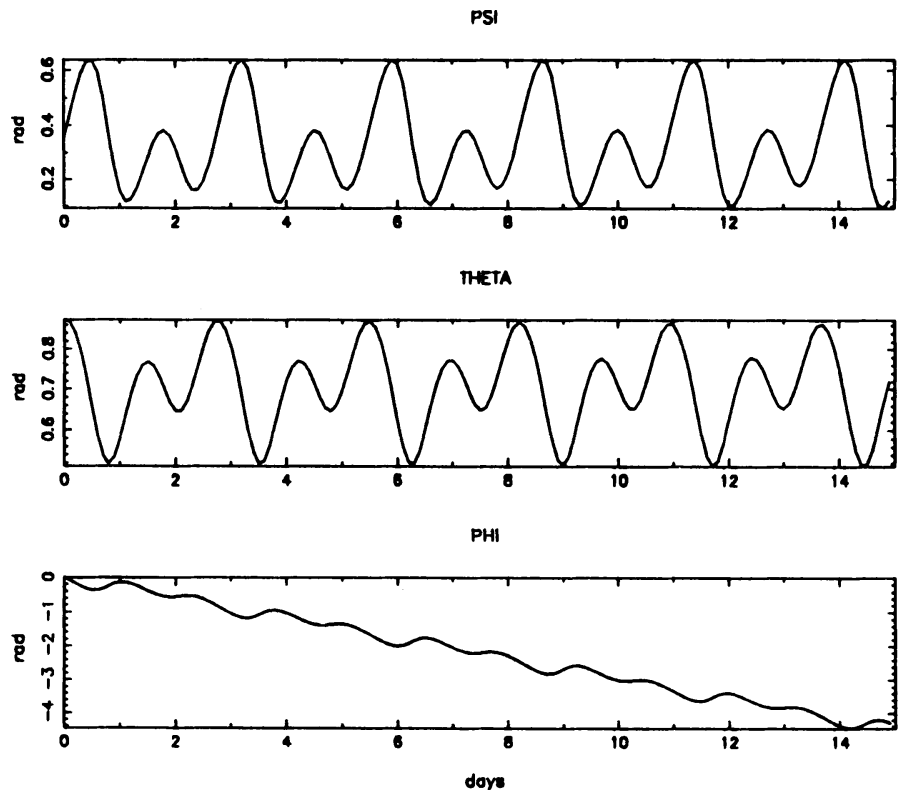

Fig. 1. Free Eulerian oscillations, shape: $8 / 4 / 3.4 \mathrm{~km}$
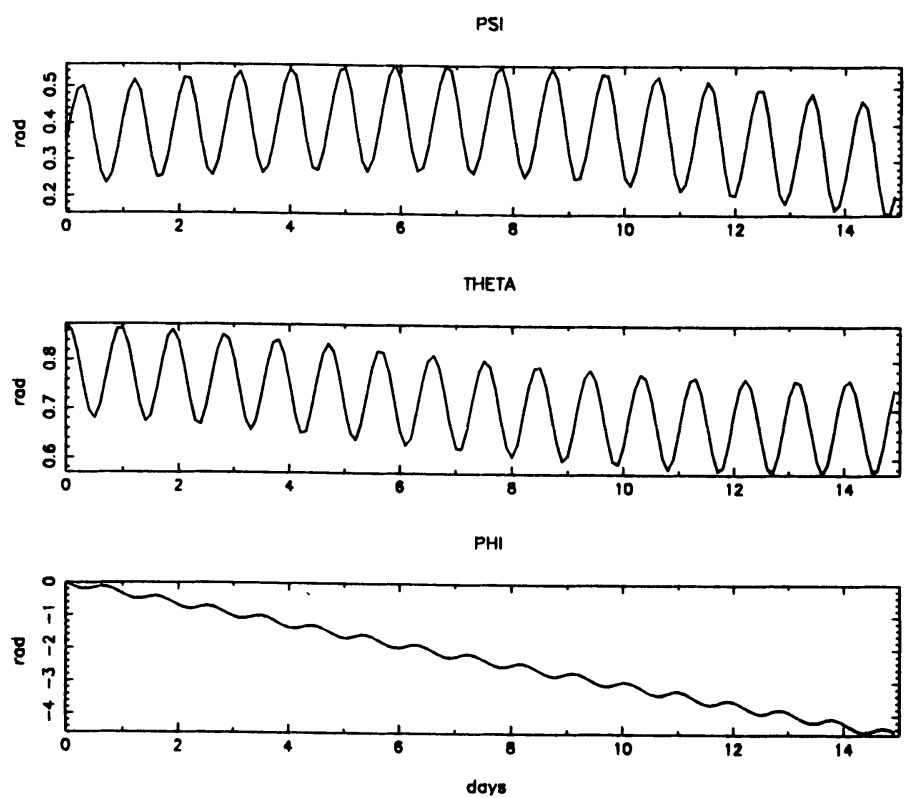

Fig. 2. Free Eulerian oscillations, shape: $10 / 5 / 1 \mathrm{~km}$ 
conditions: for both curves, the initial angles are 20,50, and $10 \mathrm{deg}$; the initial periods are 7.4, 120, and 2.2 days respectively in $\psi, \theta, \varphi$; the shape is given by $a, b$, and $c$ respectively equal to 8,4 , and $3.4 \mathrm{~km}$ in figure $1 ; 10,5$, and $1 \mathrm{~km}$ in figure 2 . The double modulation pattern is connected to the comet dynamical shape.

\section{Solar gravitational perturbation}

In the following, the solar gravitational torque is included into the model and the orbit is Halley's one. Tests are performed for the following initial orientation: 0,50 , $0 \mathrm{deg}$; only one initial period: $P_{\varphi}=2.2$ days; shape: $10,5,1 \mathrm{~km}$. The curves present the differences between non-perturbed and perturbed cases, plotted at perihelion, with identical initial conditions. Figure 3 shows the variations of the angular momentum. Librations shown on figure 4 are then due to the solar disturbing action. They are of order several hundreds of arc seconds on this 20-day simulation. The gravitational perturbations change a little bit the rotational pattern at each perihelion passage. Its action remains to be compared with non-gravitational effects $([3])$.

\section{Close-approach configuration}

The comet is assumed to be moving on an inner orbit in Jupiter's mean plane. Comet parameters are chosen in such a way that the close encounter occur near the aphelion of both the comet and Jupiter, for different minimum distances between the two bodies. The eccentricity is Halley's one.

For an encounter distance of $1 \mathrm{AU}$, Jupiter's influence is almost negligible. When the encounter occurs for $0.1 \mathrm{AU}$, only small effects can be detected on the rotational motion evolution, in contrast to the great changes on the orbital motion.

When the encounter distance is planned to occur at $0.01 \mathrm{AU}$, the comet is ejected from an almost keplerian orbit two days before the anticipated aphelion. The osculating semi-major axis undergoes a brief but strong impulse up to $38 \mathrm{AU}$ in one day, then decreases to about $10 \mathrm{AU}$. The initial value was $2.77 \mathrm{AU}$. In the same time, the osculating eccentricity comes very close to the unity and then decreases. The initial value was 0.965 . For the last three figures, angular initial values are: $\psi=0, \theta=50, \varphi=0 \mathrm{deg}$; initial periods are: $P_{\psi}=7.4, P_{\varphi}=2.2$ days. Because the close approach happens far from the Sun, the angular momentum is almost constant until 2 or 3 days before the encounter (fig. 5). One day after, a new constant pattern is achieved. Figure 6 shows clearly the pulse-shaped torque due to Jupiter at the encounter moment. After the encounter, libration amplitudes have strongly decreased (fig. 7). The rotation $\varphi$ presents a variation of its mean motion. Again, compared with the changes on the orbital motion, the variations on the rotational one are very limited. After the close encounter, the comet rotational motion keeps the new features, like new initial conditions after an impact. The motion is greatly sensitive to particular sets of initial conditions. Resulting motions can be different, depending on the particular comet orientation at the encounter moment. Then, Jupiter naturally plays the trigger role. Indeed, with quasi identical initial conditions, the global behavior can be opposite to the previous one, with 
DELTA LX

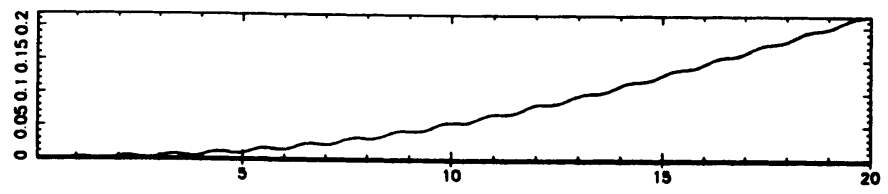

DELTA Ly
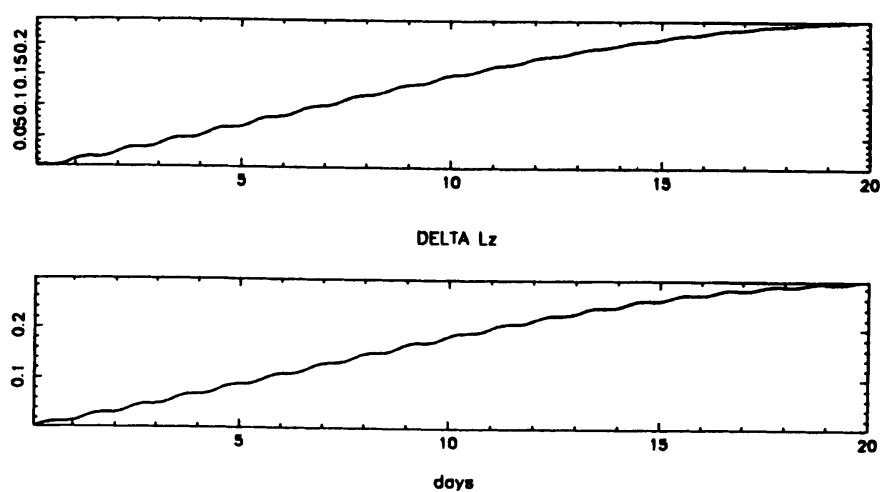

Fig. 3. Solar perturbation on the angular momentum

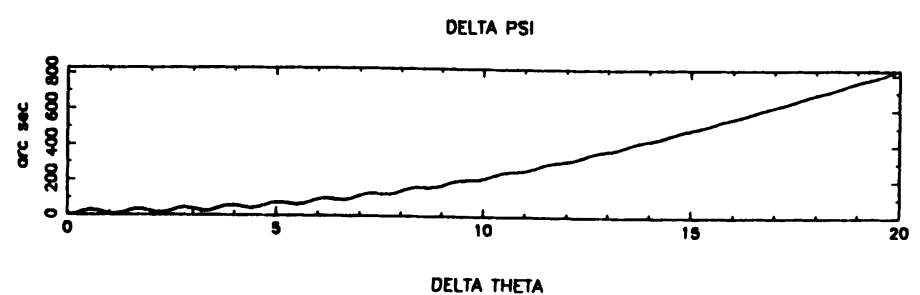

DELTA THETA

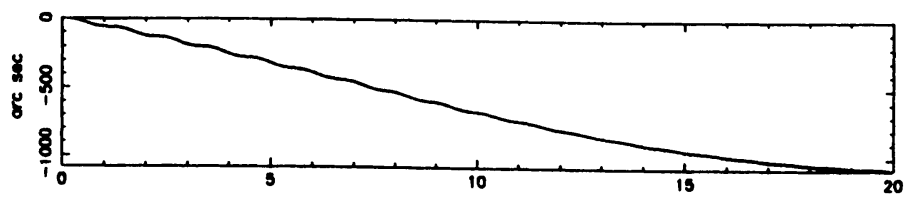

DELTA PHI

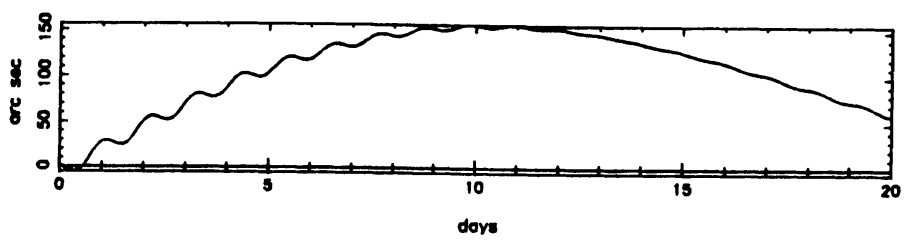

Fig. 4. Physical librations due to solar torque 

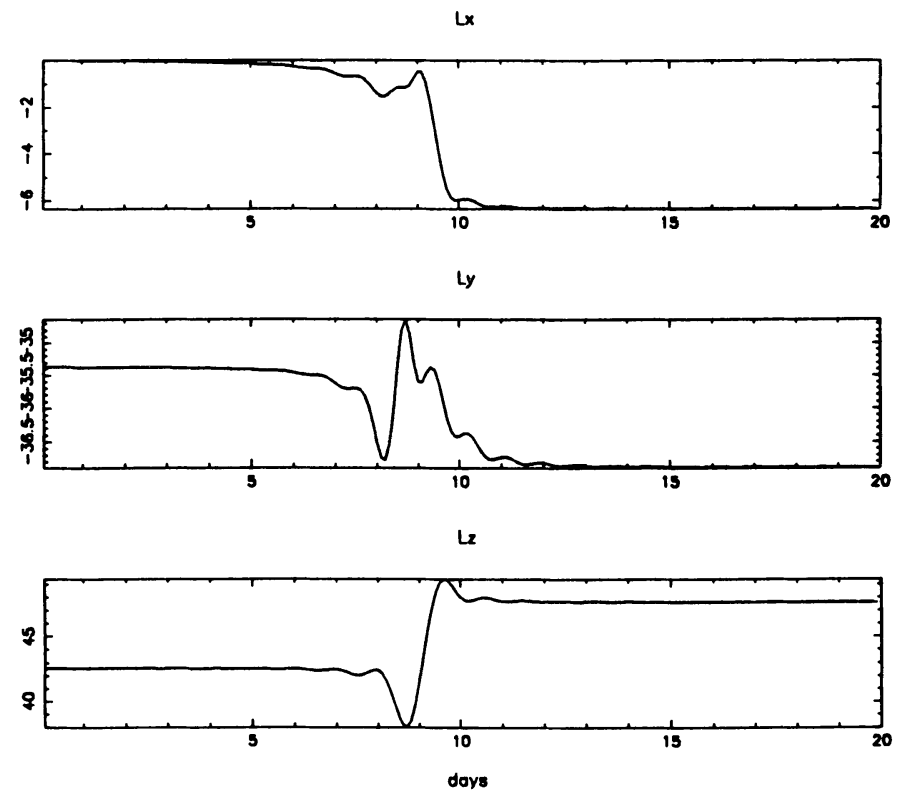

Fig. 5. Solar perturbation on the angular momentum
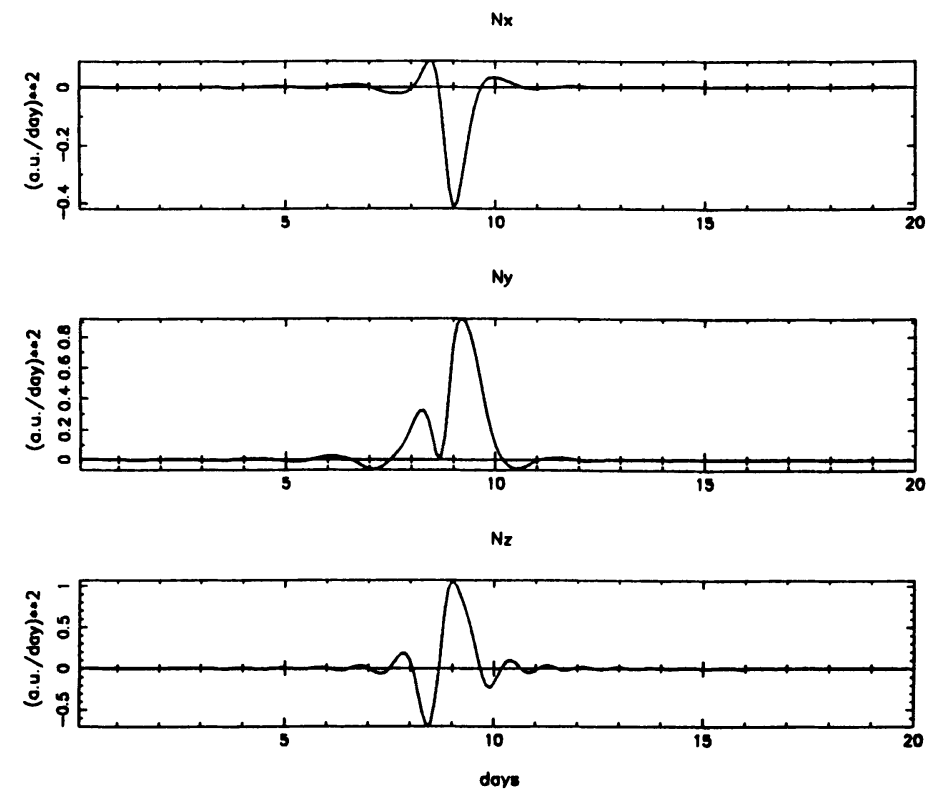

Fig. 6. Pulse shape of jovian torque 
PSI

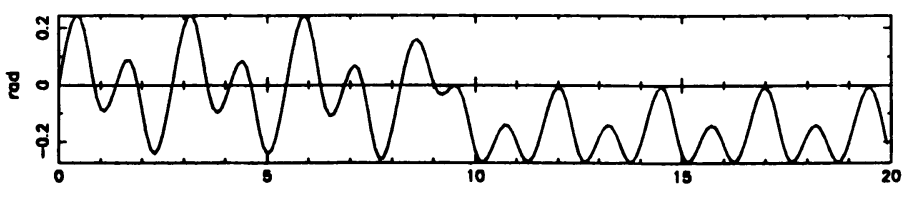

THETA

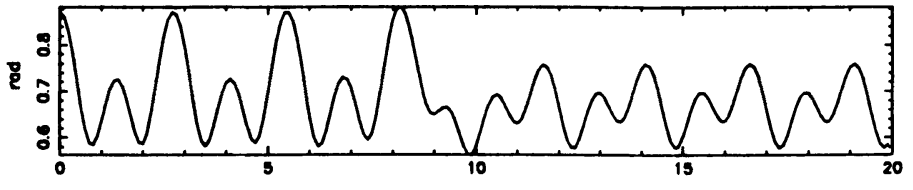

PHI

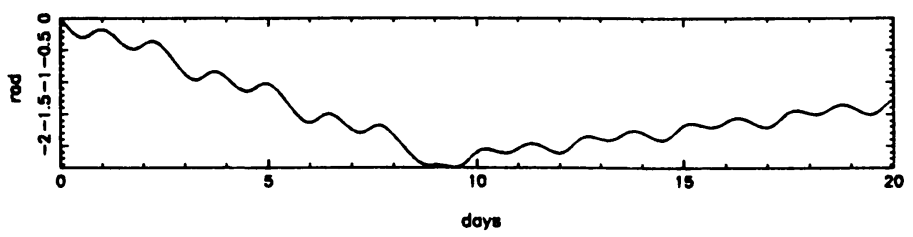

Fig. 7 . Jovian close-approach effect.

largely increased libration amplitudes.

\section{Conclusion}

Physical librations due to solar gravitational perturbations have small amplitudes with respect to simple oscillations obtained without perturbations. A close-approach with Jupiter leads to a limited change on the rotational motion compared to the orbital one. However, the motion is greatly sensitive to particular sets of initial conditions. This could lead to the idea of a possible zone of non-predictible motions in the phase space. This gravitational study has to be completed by introducing non-gravitational forces, such as surface variations and gas ejections. This next step will allow a deeper investigation of rotational behaviors in the Sun's vicinity.

\section{References}

1. Bois, E., Oberti, P., Froeschlé, C. (1991) "Gravitational Model of Comet Nucleus Rotation", submitted to Celestial Mechanics.

2. Bois, E., (1986) "First-order theory of satellite attitude motion - Application to Hipparcos", Celestial Mechanics 39, 309-327.

3. Peale, S.J., Lissauer, J.J. (1989) "Rotation of Halley's comet", Icarus 79, 396-430. 